\title{
Kinins are Generated In Vivo following Nasal Airway Challenge of Allergic Individuals with Allergen
}

\author{
David Proud, Alkis Togias, Robert M. Naclerio, Sylvia A. Crush, \\ Philip S. Norman, and Lawrence M. Lichtenstein, Division of Clinical \\ Immunology, Department of Medicine, The Johns Hopkins University School \\ of Medicine at The Good Samaritan Hospital, Baltimore, Maryland 21239
}

\begin{abstract}
A B S TRACT Using a recently developed model of nasal challenge, we have obtained data that clearly demonstrate, for the first time, kinin generation during a local allergic reaction in vivo. Allergic individuals $(n=8)$ and matched nonallergic controls $(n=8)$ were challenged intranasally with the appropriate antigen and nasal washes were taken before and after challenge. Washes were assayed for kinin, histamine, and $\left[{ }^{3} \mathrm{H}\right]-\mathrm{N}$ $\alpha$-tosyl-L-arginine methyl ester (TAME)-esterase activity. Increased kinin generation was found by radioimmunoassay (RIA) in the nasal washes of all the allergics $(5,560 \pm 1,670 \mathrm{pg} / \mathrm{ml})$ but in none of the controls $(38 \pm 16 \mathrm{pg} / \mathrm{ml})$. The presence of kinin was highly correlated with that of histamine and TAME-esterase activity and with the onset of clinical symptoms $(P<0.001)$. Serial dilutions of nasal washes produced RIA displacement curves that paralleled the standard curve, and recovery of standard kinins that were added to nasal washes was $100 \pm 4 \%(n=14)$. Kinin recovery was identical in both allergics and controls and did not vary significantly with antigen challenge. The immunoreactive kinin in nasal washes was stable to boiling and not precipitated by ethanol, but completely destroyed by carboxypeptidase B. It was evenly distributed between the sol and gel phases of nasal washes. High performance liquid chromatography analysis of the immunoreactive kinin in nasal washes showed it to be a mixture of lysylbradykinin and bradykinin. We conclude that kinins are produced during local allergic reactions in the nose and may contribute to the symptomatology of the allergic response.
\end{abstract}

This is publication No. 536 of the O'Neill Research Laboratories, The Good Samaritan Hospital, Baltimore, MD.

Received for publication 25 March 1983 and in revised form 6 July 1983.

\section{INTRODUCTION}

It is generally accepted that the pathogenesis of $\operatorname{IgE}$ mediated inflammatory processes is the result of the involvement of a variety of mediators (1). Kinins are potent local hormones that exhibit a range of pharmacological actions which have led to the suggestion that they may be important mediators of inflammatory reactions $(2,3)$. While the potential clearly exists, however, for a role of kinins in IgE-mediated events in vivo, much of the evidence to support such a role has been circumstantial. In addition, most of the existing direct evidence for kinin generation during IgE-mediated events is based on determination of blood kinin levels $(4,5)$ and must be viewed with caution in light of more recent reports indicating that earlier methodology resulted in spuriously high values (6-8).

In view of such criticism of the validity of existing data, the recent development of a reproducible and controlled system for nasal antigen challenge (9) provided us with an excellent opportunity to reexamine the potential for kinin involvement in a local allergic reaction. The pathogenesis of allergic rhinitis is presumed to involve an IgE-mediated mechanism and the present nasal challenge model has already allowed the demonstration that antigen administration leads to the release of mast cell mediators in vivo (9). We now report the first clear demonstration of kinin generation during a local allergic reaction in vivo and correlate kinin generation with the production of other mediators and with the onset of clinical symptoms.

\section{METHODS}

\section{Subjects}

Healthy male and female volunteers between the ages of 18 and 55 were recruited and they gave informed consent prior to study. Allergic individuals were defined as having 
seasonal symptoms to an antigen to which they had a positive intradermal skin reaction at a concentration of 10 protein nitrogen units $/ \mathrm{ml}$ or less of crude allergen extracts. Nonallergic individuals had no seasonal symptoms and negative skin tests to antigen extracts ( 100 protein nitrogen units $/ \mathrm{ml}$ ).

\section{Nasal challenge and sample collection}

Ragweed pollen, ragweed extract, and mixed grass extract (Greer Laboratories, Lenoir, NC) were used as allergens. Pollens were insufflated in the nose by the method of Rosenberg et al. (10). Known amounts of ragweed pollen $\left(3.18 \pm 0.2 \times 10^{8}\right.$ grains $\left./ g\right)$ were combined with lactose to obtain a total weight of $28.4 \mathrm{mg}$ and were placed in a gelatin capsule. The capsule was placed in a Spinhaler (Fisons, Bedford, MA), which was adapted for nasal application and connected to a French-Rosenthal dosimeter. When the dosimeter was activated (four times for $2 \mathrm{~s}$ with a driving pressure of $\left.10 \mathrm{lb} / \mathrm{in}^{2}\right), \sim 25 \mathrm{mg}$ of pollen and lactose $( \pm 15 \%)$ was expelled into the nose. When antigen extracts were used, they were placed in a DeVilbiss 646 nebulizer (DeVilbiss Co., Somerset, PA) which was adapted to fit into a nostril. Like the Spinhaler, it was attached to a dosimeter that allowed the delivery of $0.08 \mathrm{ml}$ of extract from the nebulizer into the nose. In the present study, individuals were given increasing doses of pollen or extract and monitored for the onset of clinical symptoms (sneezing) and for mediator output. Six individuals from each group were challenged with extract and two with pollen. There was no difference in the nature of the response to extract or pollen.

Although nasal airway resistance (NAR) ${ }^{1}$ was measured by anterior rhinometry, the unpredictable effects of washing the nose with saline on this parameter, together with the use of oxymetazoline hydrochloride (Afrin, Schering Corp., Kenilworth, NJ) to maintain nasal volume, meant that NAR could not be considered a quantitative parameter. Conductance $\left(\mathrm{NAR}^{-1}\right)$ data, however, is presented for illustrative purposes.

Nasal washes were obtained by instructing patients to tilt their heads backwards $\sim 30^{\circ}$ from the horizontal while in the sitting position, to hold their breath, and refrain from swallowing. $5 \mathrm{ml}$ of normal saline (Cutter Laboratories, Berkeley, CA) was instilled into each nostril and after $10 \mathrm{~s}$, the patients flexed their necks and expelled the mixture of mucus and saline into a collection vessel. Samples were immediately made $40 \mathrm{mM}$ in EDTA and stored on ice until the conclusion of the experiments. In some experiments, aliquots were collected in the presence and absence of EDTA for comparison of kinin values. In kinin recovery experiments, standard bradykinin was added to nasal washes immediately upon collection. In all cases, upon conclusion of the experiment, the nasal washes were centrifuged at $15,000 \mathrm{~g}$ and $4^{\circ} \mathrm{C}$ for $15 \mathrm{~min}$ and the sol phase was separated from the mucus phase. All samples were stored at $-80^{\circ} \mathrm{C}$ until assayed.

To test for the destruction of kinin in nasal washes by a known kininase, nasal washes were incubated for $5 \mathrm{~min}$ and $37^{\circ} \mathrm{C}$ with hog pancreatic carboxy peptidase B (Sigma Chemical Co., St. Louis, MO) at a final concentration of 40

\footnotetext{
${ }^{1}$ Abbreviations used in this paper: HPLC, high performance liquid chromatography; NAR, nasal airway resistance; $\mathrm{NAR}^{-1}$, conductance; PEG, polyethylene glycol, TAME, ${ }^{3} \mathrm{H}-\mathrm{N}$ - $\alpha$-tosyl-L-arginine methyl ester.
}

$\mu \mathrm{g} / \mathrm{ml}$. Samples were then boiled for $1 \mathrm{~min}$, centrifuged, and assayed either by bioassay or radioimmunoassay (RIA). Controls consisted of aliquots of the same nasal washes that were incubated with buffer and then boiled.

\section{Mediator assays}

Kinin RIA. The RIA that was used employed $\mathrm{Tyr}^{8}$-bradykinin (Peninsula Laboratories, Belmont, CA) which was iodinated by the method of Hunter and Greenwood (11). A $5-\mu \mathrm{l}(0.5 \mu \mathrm{g})$ aliquot of $\mathrm{Tyr}^{8}$-bradykinin was mixed with 30 $\mu l 0.05 \mathrm{M}$ phosphate buffer, $\mathrm{pH} 7.4$, and $5 \mu \mathrm{l}(0.5 \mathrm{mCi}) \mathrm{Na}$ ${ }^{125}$ I (Amersham Corp., Arlington Heights, IL). The reaction was initiated by the addition of $5 \mu \mathrm{l}(1 \mu \mathrm{g})$ of chloramine $\mathrm{T}$ and was allowed to proceed for $10 \mathrm{~min}$ on ice before being terminated by the addition of $10 \mu \mathrm{l}(2 \mu \mathrm{g}) \mathrm{Na}_{2} \mathrm{~S}_{2} \mathrm{O}_{5}$. Iodinated bradykinin was separated from free iodine by chromatography on a column $(0.8 \times 6 \mathrm{~cm})$ of Dowex AG $1-\mathrm{X} 4$, chloride form (Bio-Rad Laboratories, Richmond, CA). The iodinated bradykinin was diluted 1:4 with $0.2 \mathrm{M}$ Tris/0.01 M EDTA/ $0.1 \%$ lysozyme, $\mathrm{pH} 6.4$, and frozen in aliquots. The specific activity of the labeled bradykinin obtained by this method was $1.3-1.4 \mathrm{Ci} / \mu \mathrm{mol}$.

Rabbit antiserum to bradykinin was produced by multiple intradermal injections of bradykinin which had been coupled to chicken serum albumin by the method of Goodfriend et al. (12), and was a gift from Dr. John Pisano, National Heart, Lung, and Blood Institute, Bethesda, MD.

Assays were performed in $0.2 \mathrm{M}$ Tris/0.01 M EDTA/0.1\% lysozyme, $\mathrm{pH} 6.4$, and all dilutions were made in this buffer. A $200 \mu$ l volume of standard bradykinin or unknown, at suitable dilutions, was incubated with $100 \mu \mathrm{l}$ of labeled bradykinin $(10,000 \mathrm{cpm})$ and $100 \mu \mathrm{l}$ of antiserum $(1: 140,000$ final dilution) for $20-24 \mathrm{~h}$ at $4^{\circ} \mathrm{C}$. The maximum binding of labeled in the absence of nonlabeled antigen for the assay $(\sim 5,000 \mathrm{cpm})$ was determined by running tubes in which sample was replaced with buffer, while nonspecific binding was determined by using tubes containing $100 \mu \mathrm{l}$ of labeled bradykinin and $300 \mu \mathrm{l}$ of buffer. This method of determining nonspecific binding gave values identical to those obtained by using nonimmune sera or an excess of bradykinin standard and was used for convenience. Nonspecific binding in this assay was usually $\sim \mathbf{4 0 0} \mathrm{cpm}$. In all cases, antigen-antibody complex was separated from free antigen by polyethylene glycol (PEG) precipitation as follows. With all tubes in icewater, a $400 \mu$ l volume of cold $1 \%$ bovine gamma globulin (Sigma Chemical Co.) was added to each tube. This was followed by the addition of $800 \mu \mathrm{l}$ of cold 30\% PEG 8,000 (Fisher Scientific Co., Pittsburgh, PA). All tubes were vortexed and centrifuged at $3,000 \mathrm{rpm}$ for $5 \mathrm{~min}$ at $4^{\circ} \mathrm{C}$. Supernatants were removed by aspiration, and the precipitates were washed by adding $800 \mu \mathrm{l}$ of cold 15\% PEG 8,000 and vortexing. The tubes were centrifuged once more, supernatants were aspirated, and the radioactivity associated with the precipitate was counted. The bovine gamma globulin and both PEG 8,000 solutions were prepared in RIA buffer from which the lysozyme had been omitted. Samples of human urine that were collected in acid and pepstatin (13) and stored in aliquots at $-20^{\circ} \mathrm{C}$ were used as quality controls in the assay. Results were calculated using a Spline-fit computer program.

The assay was capable of detecting a minimum dose of $20 \mathrm{pg}$ bradykinin $/ \mathrm{ml}$. Inter- and intra-assay coefficients of variation $(\mathrm{SEM} /$ mean $\times 100 \%)$ are $2(n=8)$ and $5 \%$ $(n=6)$, respectively. There was an excellent correlation between the RIA and bioassay. The assay did not distinguish, on a molar basis $\left(E D_{50}=2 \times 10^{-10} \mathrm{M}\right.$ in each case), between 
the three mammalian kinins (bradykinin, lysylbradykinin, and methionyllysylbradykinin), and would also recognize other nonmammalian kinins, such as polisteskinin (14), that have the same carboxyterminus ( $\sim 30 \%$ cross-reaction). Cleavage of bonds within the kinin moiety resulted in almost complete destruction of cross-reactivity, particularly if bonds near the carboxyterminus were affected. Highly purified human kininogen displayed $<2 \%$ cross-reaction, on a molar basis, in this assay. No other cross-reacting materials are known.

Kinin bioassay. The biological activity of kinins in nasal washes was demonstrated by using isolated rat uterus as previously described (15).

Histamine. For histamine determinations, $0.8 \mathrm{ml}$ of sample was mixed with $0.2 \mathrm{ml}$ of $8 \% \mathrm{HClO}_{4}$ and centrifuged for $10 \mathrm{~min}$ at $1,000 \mathrm{~g}$. Supernatants were assayed by an automated spectrofluorometric assay (16) and concentrations of histamine of $\geq 1 \mathrm{ng} / \mathrm{ml}$ could be measured with an accuracy of $\pm 5 \%$.

$\left[{ }^{3} \mathrm{H}\right]-\mathrm{N}$ - $\alpha$-tosyl-L-arginine methyl ester (TAME)-esterase. Enzyme(s) that have arginine esterase activity were assayed essentially by the method of Imanari et al. (17), which is based on the liberation of tritiated methanol from the synthetic substrate TAME. A $40-\mu \mathrm{l}$ aliquot of nasal sample and $10 \mu \mathrm{l}$ of $0.2 \mathrm{M}$ Tris buffer, $\mathrm{pH} 8.0$, were added to a 1.5-ml eppendorf microtube. A $10 \mu \mathrm{l}$ volume $\left(\sim 1.5 \times 10^{5} \mathrm{dpm}\right)$ of $\left[{ }^{3} \mathrm{H}\right]-\mathrm{TAME}$ (Amersham Corp.) was then added with mixing and the tube was placed in a counting vial containing $10 \mathrm{ml}$ of Econofluor (New England Nuclear, Boston, MA) and $50 \mu \mathrm{l}$ of stop solution ( 1 vol glacial acetic acid $/ 9 \mathrm{vol} 0.02 \mathrm{M}$ TAME). The counting vial was then tightly capped and after $1 \mathrm{~h}$, the reaction was terminated and the tritiated methanol was partitioned into the Econofluor by shaking. The vial was then counted for 4 min in a liquid scintillation spectrometer.

\section{Ethanol extractions}

To test the stability of the nasal kinin to ethanol precipitation, and to compare the kinin content of the sol and gel phase of nasal washes, the following procedure was used. Nasal samples, with and without added standard kinin, were separated into sol and gel phases by centrifugation as described above, and equal amounts of each phase (determined by both weight and volume) were used. $4 \mathrm{ml}$ of $95 \%$ ethanol was mixed with $1 \mathrm{ml}$ of sample (either sol or gel) and left on ice for $15 \mathrm{~min}$. The mixture was then centrifuged at 1,000 $g$ for $10 \mathrm{~min}$ and the supernatant was removed while the sediment was washed with $0.5 \mathrm{ml}$ of $80 \%$ ethanol and the centrifugation repeated. Both supernatants were combined and vacuum-extracted to dryness. The sample was reconstituted to $1 \mathrm{ml}$ with $0.2 \mathrm{M}$ Tris/0.01 M EDTA/0.1\% lysozyme, $\mathrm{pH} 6.4$, and assayed as described above.

\section{High performance liquid chromatography (HPLC) analysis}

Kinin-containing nasal washes from an individual were pooled and prepared for analysis by passage over Sep-Pak $\mathrm{C}_{18}$ cartridges (Waters Associates, Milford, MA). After washing with $0.05 \%$ trifluoroacetic acid $/ 0.025 \%$ triethylamine, pH 2.5 (solution A), kinins were eluted from the cartridge by using $50 \%$ solution $A / 50 \%$ solution $B$ (solution B: $80 \%$ acetonitrile in solution $A$ ) and concentrated to 50-100 $\mu$ l by using a Savant Speed Vac concentrator (Savant Instruments, Inc., Hicksville, NY). Recovery of $\left[{ }^{3} \mathrm{H}\right]$ bradykinin by this procedure was $90 \%$. Samples were centrifuged at $10,000 \mathrm{~g}$ for $2 \mathrm{~min}$ to remove any particulate material before application to the column. To ensure that there was no carry-over of standard kinins into the nasal samples, the sample loop was washed with acetonitrile after each run and a negativecontrol run was performed before the application of the nasal samples.

Kinins were separated by using a Beckman Model 324 liquid chromatograph equipped with a $3.9 \times 300 \mathrm{~mm} \mu$ Bondapak $\mathrm{C}_{18}$ column of $10-\mu \mathrm{m}$ particle size (Waters Associates). Separation was performed under isocratic conditions at a flow rate of $1 \mathrm{ml} / \mathrm{min}$ with a mobile phase consisting of $75 \%$ solution $\mathrm{A} / 25 \%$ solution $\mathrm{B}$. Absorbance was monitored at $210 \mathrm{~nm}$ and $1 \mathrm{ml}$ fractions were collected. Fractions were concentrated to 50-100 $\mu \mathrm{l}$ by using the Savant concentrator and reconstituted to $1 \mathrm{ml}$ with $0.2 \mathrm{M}$ Tris/0.01 M EDTA/ $0.1 \%$ lysozyme, $\mathrm{pH} 6.4$, for assay as described above.

When standard kinins were added to control nasal washes and put through the Sep-Pak extraction and HPLC procedure, final recoveries were $84 \pm 11$ for bradykinin, $74 \pm 7$ for lysylbradykinin, and $84 \pm 8 \%$ for methionyllysylbradykinin ( $n$ $=3$ in each case). The minimum amount of each kinin which could be detected by $\mathrm{A}_{210}$ was $\sim 30 \mathrm{ng}$ for bradykinin and $20 \mathrm{ng}$ each for lysylbradykinin and methionyllysylbradykinin.

\section{RESULTS}

The experimental protocol used and representative results for a nonallergic control and an allergic individual are shown in Figs. 1 and 2, respectively. Individuals from both experimental groups frequently had significant levels of mediators in the resting state. To ensure a low, stable base line, four washes were performed. Oxymetazoline hydrochloride was then administered

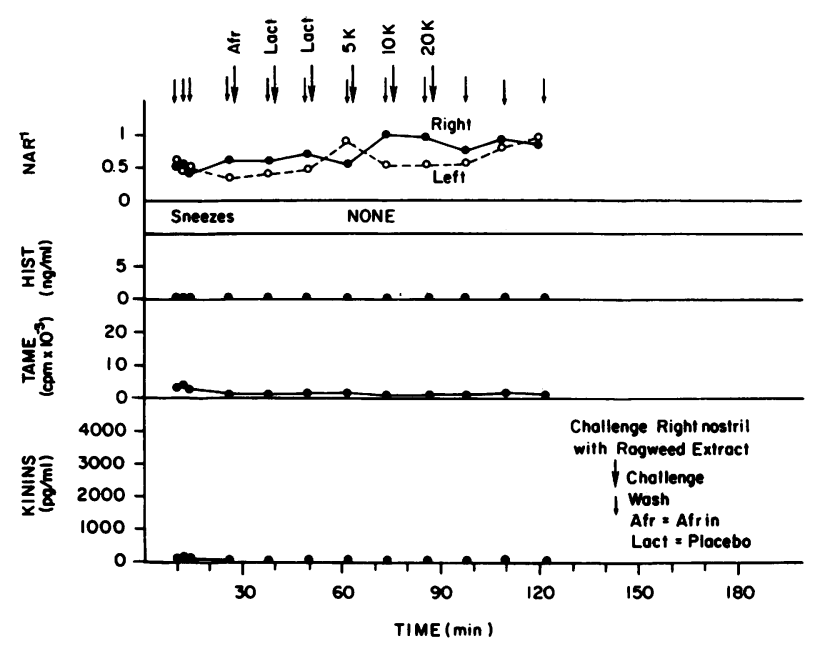

Figure 1 Nasal challenge of a nonallergic control. The experimental protocol is shown at the top of the figure. Smaller arrow indicates saline wash and larger arrow indicates administration of the material indicated. Ragweed extract dosages are in thousands of protein nitrogen units per milliliter (K). NAR ${ }^{-1}$ is nasal conductance expressed in liters per second per centimeter $\mathrm{H}_{2} \mathrm{O}$. Hist, histamine; lact., lactose. 


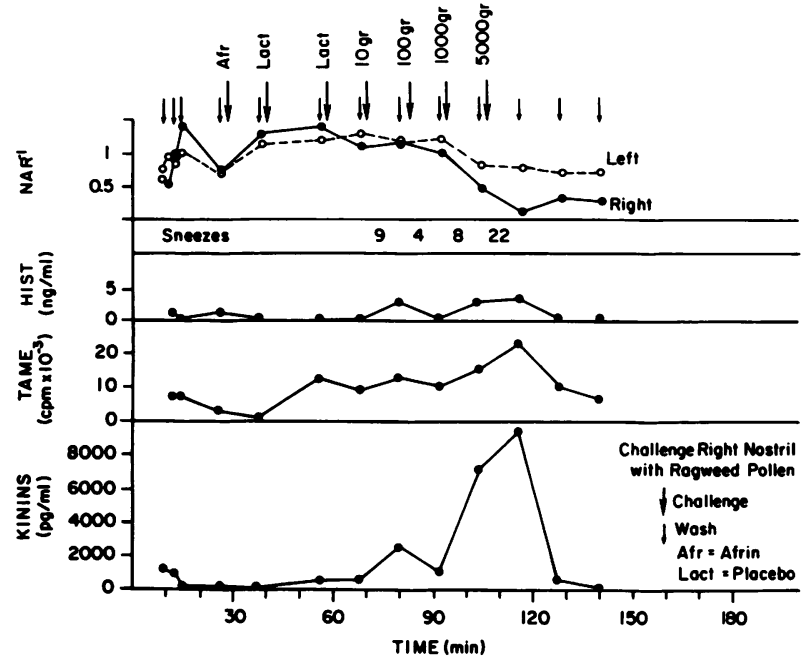

Figure 2 Nasal challenge of an allergic individual. Ragweed pollen dosages are in grains (gr). Hist, histamine; lact., lactose.

and an additional wash was performed 10 min later. Two administrations of lactose were given as controls and then increasing doses of antigen were insufflated. Nasal washes were performed at fixed time intervals after administration of placebo or antigen. As seen in Fig. 1, the control individual did not produce any mediators or show clinical symptoms at any level of antigen used. In contrast, the allergic individual (Fig. 2), while showing no response to placebo challenge, produced kinins in a dose-dependent response to antigen challenge. Generation of kinin was accompanied by the production of histamine and TAME-esterase activity, and was associated with the onset of clinical symptoms as indicated by the onset of sneezing. At the higher doses of antigen used, there was a clear decrease in nasal airway conductance despite the earlier administration of oxymetazoline. The apparent production of TAME-esterase that was seen following the first placebo challenge of this individual was not representative of other experiments. The lack of production of other mediators at this point suggested an aberrant TAME-esterase value.

Since kinins are known to be easily destroyed in vivo (18), early experiments $(n=5)$ were performed by collecting nasal washes in the presence and absence of EDTA (an inhibitor of some of the major human kininases). Typical results are shown in Fig. 3. While the qualitative response to antigen challenge was identical in both cases, the maximum kinin response was threefold higher in the presence of EDTA; this indicated the presence of kininase activity in nasal secretions. All experiments were, therefore, performed by collecting nasal washes in EDTA.

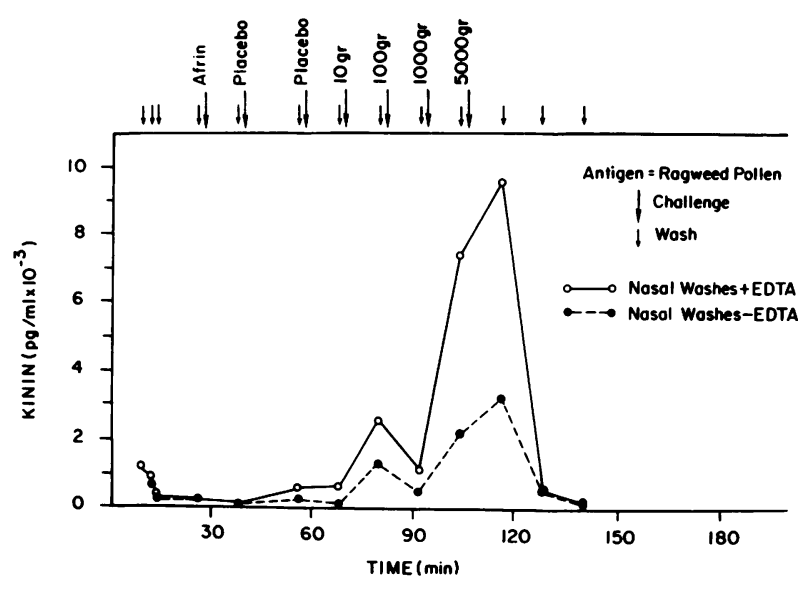

Figure 3 Collection of nasal washes in the presence and absence of EDTA.

Before performing experiments on matched allergics and nonallergic controls, it was necessary to demonstrate that the RIA could provide accurate quantitative measurements. This was done by showing that serial dilutions of nasal samples produced a RIA displacement curve that paralleled the standard curve (Fig. 4). In addition, it was shown that recovery of standard kinins that were added to nasal washes at collection was $101 \pm 3 \%$ ( $\overline{\mathrm{x}} \pm \mathrm{SEM} ; \boldsymbol{n}=11$ ) for control individuals and $100 \pm 4 \%(n=14)$ for allergics. Recovery of added kinins showed no significant variation at any stage in the experimental protocol. It was also shown, by ethanol extraction of mucus, that immunoreactive kinin was evenly distributed between sol and gel phases of the nasal washes; therefore, the sol phase was used for all further assays.

The kinin data obtained after antigenic challenge of

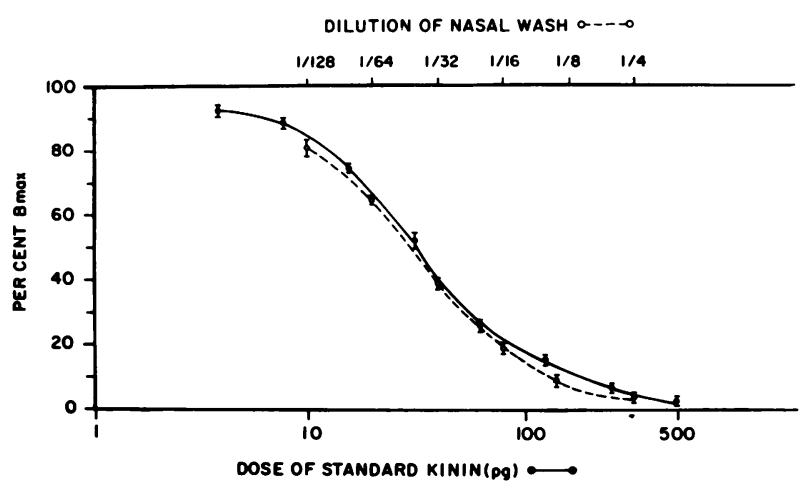

Figure 4 Parallel cross-reaction of nasal washes in the kinin RIA. Serial dilutions of a postchallenge nasal wash containing immunoreactive kinin were compared with the standard curve. $B_{\max }$, maximum binding in absence of nonlabeled antigen. 
TABLE I

Kinin Production in Response to Intranasal Antigen Challenge

\begin{tabular}{|c|c|c|c|}
\hline \multicolumn{2}{|c|}{ Controls } & \multicolumn{2}{|c|}{ Allergics } \\
\hline Base line & Response & Base line & Response \\
\hline \multicolumn{2}{|c|}{$p g / m l$} & \multicolumn{2}{|c|}{$p g / m l$} \\
\hline$<20$ & $<20$ & $<20$ & 520 \\
\hline$<20$ & $<20$ & $<20$ & 5,000 \\
\hline$<20$ & $<20$ & $<20$ & 1,830 \\
\hline$<20$ & $<20$ & 130 & 9,950 \\
\hline 100 & 150 & 600 & 9,925 \\
\hline$<20$ & $<20$ & $<20$ & 580 \\
\hline$<20$ & $<20$ & 200 & 12,900 \\
\hline$<20$ & 40 & 60 & 4,140 \\
\hline \multicolumn{2}{|c|}{$\overline{\mathrm{x}} \pm \mathrm{SEM}=38.5 \pm 16$} & \multicolumn{2}{|c|}{$\overline{\mathrm{x}} \pm \mathrm{SEM}=5,560 \pm 1,666$} \\
\hline
\end{tabular}

Base-line values are those in the last placebo wash. Response values indicate the maximum kinin concentration observed after antigenic challenge.

eight allergic individuals and eight nonallergic controls is summarized in Table I. Base-line values refer to those after the last placebo challenges while response values refer to the maximum amount of kinin generated in postantigen challenges washes. Dramatic increases in kinin were seen after antigen challenge of allergic patients, but no significant kinin generation occurred with nonallergic controls. A similar picture was seen for
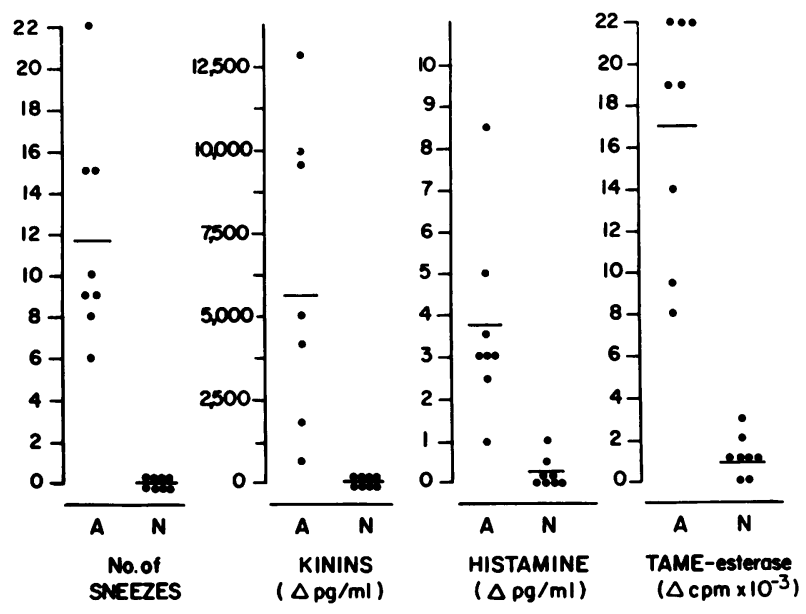

\section{$A=A L L E R G I C$ \\ $N=$ NONALLERGIC}

FIGURE 5 A comparison of kinin generation, sneezing, and other mediators in eight matched allergic $(A)$ and nonallergic $(N)$ subjects. The dots represent the maximum number of sneezes or the maximum concentration of mediator in the nasal wash of each subject. The arithmetic means for each parameter are represented by horizontal lines. other mediators and for sneezing (Fig. 5). By Wilcoxon paired analysis, sneezing and the concentrations of kinins, histamine, and TAME-esterase activity were all significantly elevated in allergics challenged with antigen as compared with nonallergics $(P<0.001)$.

No fixed ratio was observed for the levels of various mediators produced by an individual. However, if mediator release is defined as positive when a threefold increase occurred after antigen challenge as compared with levels after the last placebo challenge, and sneezing is defined as positive when it occurred three or more times after antigen administration, then kinin generation was highly correlated with sneezing and the production of each of the other mediators $(P<0.001)$ by Fischer's exact test. No positive response was observed for a parameter in any nonallergic control.

To clearly define that the immunoreactive material detected by RIA was truly kinin, a variety of parameters, in addition to immunological identity and linear addition of standard bradykinin, were used. The immunoreactive kinin was stable to boiling and was not precipitated by ethanol, but was completely destroyed $(n=3)$ by incubation with carboxypeptidase B, the most potent kininase known (18). In addition, those nasal washes with high levels of immunoreactive material were shown to produce contraction of the isolated rat uterus $(n=3)$. This ability to contract the uterus was also abolished by carboxypeptidase B $(n=3)$.

HPLC analysis of the immunoreactive kinin in nasal washes is shown in Fig. 6. The conditions used enabled separation of standard lysylbradykinin and bradykinin by a difference of $5.5 \mathrm{~min}$ in retention time. Standard methionyllysylbradykinin (not shown) had a retention 1.5 min longer than that of bradykinin. Application of varying levels of the three standard kinins showed that a similar recovery of immunoreactive material was observed for each of the three kinins $\mathbf{7 3} \pm 1$ for bradykinin, $76 \pm 3$ for lysylbradykinin, and $76 \pm 1 \%$ for methionyllysylbradykinin; $n=3$ in each case). An excellent correlation between $A_{210}$ and recovered immunoreactivity was observed for each kinin. Buffer control runs that were performed immediately after chromatography of the standards showed little or no immunoreactivity. In contrast, chromatography of a nasal sample (Fig. $6 \mathrm{C}$ ) revealed two peaks of immunoreactivity with retention times that corresponded exactly to those of lysylbradykinin and bradykinin. In the case of the patient shown, lysylbradykinin represented $25 \%$ of the total immunoreactivity recovered. A second patient (not shown) also showed two peaks of immunoreactivity with lysylbradykinin representing $34 \%$ of the total kinin detected. In both cases, methionyllysylbradykinin was not detected. Since recoveries of all three kinins from control washes were comparable, the above results cannot 

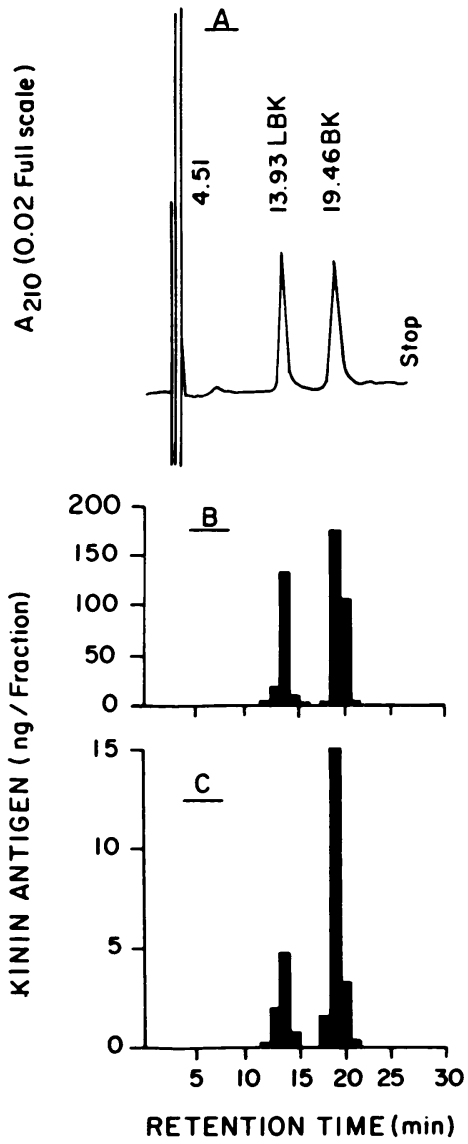

FIGURE 6 HPLC analysis of nasal kinin extract. The analysis is performed under isocratic conditions as described in the text. (A) Absorbance profile observed upon application of $300 \mathrm{ng}$ of bradykinin and $200 \mathrm{ng}$ of lysylbradykinin. LBK, lysylbradykinin; BK, bradykinin. (B) Immunoreactive kinin in fractions corresponding to the UV absorbing peaks. $(C)$ Immunoreactive kinin in fractions collected during chromatography of nasal kinin extract.

be explained on the basis of selectively lower recoveries of lysylbradykinin and methionyllysylbradykinin.

\section{DISCUSSION}

The known ability of kinins to produce all the cardinal signs of inflammation, including vasodilatation, increased vascular permeability, and edema, makes them prime candidates as important mediators of IgE-mediated inflammatory events. The recent description of proteases that are released from human mast cells by IgE-mediated mechanisms (19-21) and that are capable of interacting with the kinin system and the Hageman factor-dependent pathways provided the first clear rationale to explain kinin formation during the allergic response. However, despite the report that high molecular weight kininogen is consumed during human anaphylaxis (22), there has been little direct evidence of in vivo kinin formation during allergic reactions and attempts at blood kinin measurements have been complicated by technical difficulties and by the short survival time of these peptides in the circulation. In light of these problems, we decided to focus on an alternate in vivo system.

The nasal airways are readily accessible and samples may be obtained by relatively noninvasive techniques. In spite of these advantages, there have been few attempts to study mediator release in the nose and these, by and large, have not been definitive. The only previous report on the existence of kinin-like activity in the nasal secretions of allergic patients (23) was limited by the inability to obtain adequate samples from nonallergic controls or from allergic subjects prior to challenge. In addition, kinin activity was found in only half of the allergics tested after challenge and no attempt was made to identify the kinin-like material.

The present results, on the other hand, show a clear relationship between the generation of kinin and the onset of clinical symptoms observed after antigen challenge. In addition, kinin production was highly correlated with the appearance of histamine and TAMEesterase activity. Whether the antigenic challenge procedure used in the present study adequately reflects field exposure is uncertain, but it should be emphasized that several patients in the present study, as well as numerous others studied since, responded when only 10 grains of pollen were blown into the nose. The level of kinin response seen in the present studies was dramatic; there were increases of up to 250 -fold that seen in the last placebo wash. To our knowledge, no other system has been studied which showed such increases. Since the kinin-generating ability in blood is considerable, an obvious concern was whether sneezing, which was induced by antigen challenge, resulted in blood contamination in nasal washes as a result of the rupture of blood vessels. In none of the individuals studied did we find any evidence of blood contamination and nasal washes obtained from allergic individuals who sneezed spontaneously, or as a result of mechanical stimulation with tissue paper, contained no detectable levels of kinins. In addition, it is unlikely that the parallel RIA displacement curves and excellent recoveries of added kinin would be observed in the presence of blood contamination. Finally, the presence of considerable amounts of lysylbradykinin in nasal washes cannot be explained purely on the basis of activation of the plasma system because plasma kallikrein produces only bradykinin (24). The failure to detect methionyllyslbradykinin in the present studies was not particularly surprising. This peptide has, to date, only been detected 
in acidified plasma (25) or urine (26) and is probably not normally present in vivo.

The occurrence of both lysylbradykinin and bradykinin in nasal washes raised the possibility that several enzyme systems may be involved in kinin production. The nasal allergic response is presumed to involve the release of mast cell mediators. Of the mediators detected to date, only histamine is limited strictly to mast cells and basophils. There is also some evidence, however, that much of the TAME-esterase activity detected in nasal washes (9) resembles the major neutral protease described from human mast cells (27), and prostaglandin $D_{2}$, which has also been detected (9), is the major cyclooxygenase product of human mast cells. Since kininogenase activity has been detected in human mast cells (19) and basophils (28), it is an attractive possibility that these enzymes may contribute to kinin generation in the nose. Alternatively, mast cell proteases capable of activating the Hageman factor-dependent pathways $(20,21)$ may play a role in the activation of plasma kallikrein that diffuses into the nasal mucosa, or Hageman factor may be activated as a result of damage to the mucosa or submucosal tissue. A possible involvement of the glandular kallikrein system must also be considered since salivary kallikrein is released by sympathetic nerve stimulation $(29,30)$ and kallikrein-like activity, perhaps from salivary glands in the nose, has been detected in nasal secretions of the cat upon stimulation of the vidian nerve (31). Finally, the possibility exists that one enzyme system is responsible for the generation of lysylbradykinin, and that bradykinin may be formed as a result of the action of a kinin-converting aminopeptidase, such as the one described from human serum (32).

In summary, we have presented the first clear demonstration of kinin generation during a local allergic reaction in vivo. Nasal washes can be assayed for kinin directly, yielding reliable quantitative estimates and kinin generation was highly correlated with clinical symptoms and the generation of other mediators. The kinin generated in response to antigenic challenge was a mixture of lysylbradykinin and bradykinin. While the exact means of kinin production awaits delineation, we conclude that kinins are produced during the allergic response and may contribute to the symptomatology of the allergic reaction.

\section{ACKNOWLEDGMENTS}

The authors extend their thanks to Dr. Stephen P. Peters and Dr. John J. Pisano for useful discussions and assistance with the HPLC analysis of kinins.

This work was supported by National Institutes of Health grants HL-24210, AI-04866, and AI-08270.

\section{REFERENCES}

1. Wintroub, B. U. 1980. Inflammation and mediators. Int. J. Dermatol. 19:436-442.

2. Lewis, G. P. 1970. Kinins in inflammation and tissue injury. In Handbook of Experimental Pharmacology. E. G. Erdos, editor. Springer-Verlag, Berlin. 25:516-530.

3. Garcia Leme, J. 1978. Bradykinin-system. Handbook of Experimental Pharmacology. J. R. Vane and S. H. Ferreira, editors. Springer-Verlag, Berlin. 50/I:464-522.

4. Brocklehurst, W. E. 1968. Kinin and kinin-forming enzymes in anaphylaxis. In Biochemistry of the Acute Allergic Reactions. K. F. Austen and E. L. Becker, editors. Blackwell Publisher Ltd., Oxford. 297-303.

5. Abe, K., N. Watanabe, N. Kumagai, T. Mouri, T. Seki, and $\mathrm{K}$. Yoshinaga. 1967. Circulating plasma kinin in patients with bronchial asthma. Experientia (Basel). 23:626-627.

6. Mashford, M. L., and M. L. Roberts. 1972. Determination of blood kinin levels by radioimmunoassay. Biochem. Pharmacol. 21:2727-2735.

7. Hulthen, U. L., and T. Borge. 1976. Determination of bradykinin in blood by a sensitive radioimmunoassay. Scand. J. Clin. Lab. Invest. 36:833-839.

8. Scicli, A. G., T. Mindroiu, G. Scicli, and O. A. Carretero. 1982. Blood kinins, their concentration in normal subjects and in patients with congenital deficiency in plasma prekallikrein and kininogen. J. Lab. Clin. Med. 100:81-93.

9. Naclerio, R. M., H. L. Meier, A. Kagey-Sobotka, N. F. Adkinson, Jr., D. A. Meyers, P. S. Norman, and L. M. Lichtenstein. Mediator release after nasal airway challenge with allergen. Am. Rev. Respir. Dis. In Press.

10. Rosenberg, G. L., R. R. Rosenthal, and P. S. Norman. 1983. Inhalation challenge with ragweed pollen in ragweed-sensitive asthmatics. J. Allergy Clin. Immunol. 71:302-310

11. Hunter, W. M., and F. C. Greenwood. 1962. Preparation of ${ }^{131}$ I labelled human growth hormone of high specific activity. Nature (Lond.). 194:495-496.

12. Goodfriend, T. L., L. Levine, and G. D. Fasman. 1964. Antibodies to bradykinin and angiotensin: a use of carbodiimides in immunology. Science (Wash. DC). 144:1344-1346.

13. Proud, D., M. Perkins, J. V. Pierce, K. N. Yates, P. F. Highet, P. L. Herring, M. Mangkornkanok/Mark, R. Bahu, F. A. Carone, and J. J. Pisano. 1981. Characterization and localization of human renal kininogen. $J$. Biol. Chem. 256:10634-10639.

14. Pisano, J. J. 1968. Vasoactive peptides in venoms. Fed. Proc. 27:58-62.

15. Proud, D., J. V. Pierce, and J. J. Pisano. 1980. Radioimmunoassay of human high molecular weight kininogen in normal and deficient plasmas. J. Lab. Clin. Med. 95:563-574.

16. Siraganian, R. 1971. An automated continuous flow system for the extraction and fluorometric analysis of histamine. Anal. Biochem. 57:283-294.

17. Imanari, T., T. Kaizu, H. Yoshida, K. Yates, J. V. Pierce, and J. J. Pisano. 1976. Radiochemical assays for human urinary, salivary, and plasma kallikreins. In Chemistry and Biology of the Kallikrein-Kinin System in Health and Disease. J. J. Pisano and K. F. Austen, editors. Department of Health, Education, and Welfare Publ. No. (NIH) 76-791, Washington, DC. 205-213. 
18. Erdos, E. G., and H. Y. T. Yang. 1970. Kininases. In Handbook of Experimental Pharmacology. E. G. Erdos, editor. Springer-Verlag, Berlin. 25:289-323.

19. Proud, D., E. S. Schulman, D. W. MacGlashan, J. V. Pierce, and H. H. Newball. 1982. Anaphylactic release of a kininogenase from purified human lung mast cells. Clin. Res. 30:165A. (Abstr.)

20. Newball, H. H., H. L. Meier, A. P. Kaplan, S. D. Revak, C. G. Cochrane, and L. M. Lichtenstein. 1981. Activation of Hageman factor by proteases released during antigen challenge of human lung. Trans. Assoc. Am. Physicians. 94:126-134.

21. Meier, H. L., B. Flowers, M. Silverberg, A. P. Kaplan, D. W. MacGlashan, E. S. Schulman, and H. H. Newball. 1982. Localization and characterization of lung Hageman factor activator. Fed. Proc. 41:377 (Abstr.)

22. Smith, P. L., A. Kagey-Sobotka, E. R. Bleecker, R. Traystman, A. P. Kaplan, H. Gralnick, M. D. Valentine, S. Permutt, and L. M. Lichtenstein. 1980. Physiologic manifestations of human anaphylaxis. J. Clin. Invest. 66:1072-1080.

23. Dolovich, J., N. Back, and C. E. Arbesman. 1970. Kininlike activity in nasal secretions of allergic patients. Int. Arch. Allergy. 38:337-344.

24. Webster, M. E., and J. V. Pierce. 1963. The nature of the kallidins released from human plasma by kallikreins and other enzymes. Ann. NY Acad. Sci. 104:91-107.

25. Elliott, D. F., and Lewis, G. P. 1965. Methionyl-lysyl- bradykinin, a new kinin from ox blood. Biochem. J. 95:437-447.

26. Hial, V., H. R. Keiser, and J. J. Pisano. 1976. Origin and content of methionyl-lysyl-bradykinin, lysyl-bradykinin and bradykinin in human urine. Biochem. Pharmacol. 25:2499-2503.

27. Schwartz, L. B., R. A. Lewis, and K. F. Austen. 1981. Tryptase from human pulmonary mast cells. Purification and characterization. J. Biol. Chem. 256:1193911943.

28. Newball, H. H., R. W. Berninger, R. C. Talamo, and L. M. Lichtenstein. 1979. Anaphylactic release of a basophil kallikrein-like activity. I. Purification and characterization. J. Clin. Invest. 64:457-465.

29. Barton, S., E. J. Sanders, M. Schachter, and M. Uddin. 1975. Autonomic nerve stimulation, kallikrein content and acinar cell granules of the cats submandibular gland. J. Physiol. (Lond.). 251:363-369.

30. Maranda, B., J. A. A. Rodrigues, M. Schachter, T. K. Shnitka, and J. Weinberg. 1978. Studies on kallikrein in the duct systems of the salivary glands of the cat. $J$. Physiol. (Lond.). 276:321-328.

31. Eccles, R., and H. Wilson. 1973. A kallikrein-like substance in cat nasal secretion. Br. J. Pharmacol. 49:712714.

32. Guimaraes, J. A., D. R. Borges, E. S. Prado, and J. L. Prado. 1973. Kinin-converting aminopeptidase from human serum. Biochem. Pharmacol. 22:3157-3172. 